\title{
Retrospective chart- review of tetanus cases admitted in a tertiary care hospital
}

\author{
Bhupendra Shah', Manish Subedi', Bijay Bartaula', Ashish Ghimire² \\ 'Department of Internal Medicine, B.P.Koirala Institute of Health science \\ ${ }^{2}$ Department of Anaesthesiology and critical care, B.P.Koirala Institute of Health science
}

Keywords: infectious disease; tropical neurology; risk factors; outcome

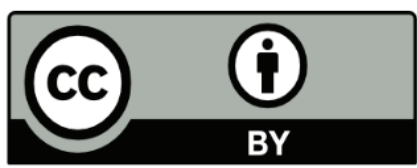

This work is licensed under a Creative Commons Attribution 4.0 Unported License.

\section{Abstract}

Background and Aims: Tetanus is a cause of thousands of death and disability annually in developing countries. Nepal is a low-income country and has achieved maternal-neonatal transmission elimination status. However, the literature regarding the status of generalized tetanus in adult populations is limited. This study was conducted to determine the clinical profile and outcome of patients with generalized tetanus.

Methods: This was a retrospective study conducted among adult patients with generalized tetanus admitted at the Department of Internal Medicine or Department of Anesthesiology and Critical Care at B.P. Koirala Institute of Health Sciences, Dharan, Nepal from January 2012 to December 2016.

Results: Of 40 patients with generalized tetanus, the mean age was $47 \pm 16$ years. Most of the patients were male $(95 \%)$ and were not vaccinated with tetanus toxoid $(67.5 \%)$. The most common wound type was skin puncture. Tracheostomy was done in 30 patients (75\%). Twenty-nine patients $(72.5 \%)$ were discharged after completion of treatment, seven patients $(17.5 \%)$ took leave against medical advice, and three patients $(7.5 \%)$ died at the hospital during treatment. Conclusions: Generalized tetanus is still a public health concern in Nepal. It is more common in males and at ages above 40 years. Perforative skin wounds sustained from the wood sticks or needles were a frequent cause. Most of the patients with generalized tetanus needed a tracheostomy and mechanical ventilation. The in-hospital mortality rate of generalized tetanus was $7.5 \%$.

the available supportive system..$^{5-6}$ The major causes of mortality are aspiration pneumonia, laryngospasm, and respiratory failure. ${ }^{7}$ Predictors of mortality are rapid progression, advanced age, and severe symptoms. ${ }^{8}$

An extensive immunization program was started in 1979 in Nepal, and maternal neonatal tetanus has been eliminated since 2005. ${ }^{9}$ Despite this progress, the adult population born before 1979 is still largely not immunized against tetanus and remains at risk. Here, we conducted a retrospective study to determine the clinical profile and outcomes of patients with generalized tetanus

*Corresponding Author:

Dr Bhupendra Shah

Assistant Professor, Department of Internal Medicine

B.P.Koirala Institute of Health Sciences, Dharan, Nepal

Email: bhupendra.shah@bpkihs.edu, doctorbhupen@gmail.com 
admitted in Department of Internal Medicine or Department of Anesthesiology and critical care at the B.P. Koirala Institute of Health Sciences (BPKIHS), which is a tertiary care center in Eastern Nepal with a catchment area of sixteen districts in Eastern Nepal and bordering areas of Southern India. ${ }^{10}$

\section{METHODS}

\section{Study design}

This was a retrospective study conducted at BPKIHS. Patients of 18 years and above with clinician made diagnoses of generalized tetanus, and admitted to the Department of Internal Medicine or Department of Anesthesiology and Critical care from January 2012 to December 2016 were enrolled as cases for this study.

\section{Ethics Statement}

This study received an ethical review from an Institutional Review Board of BPKIHS, Dharan, Nepal (Reference no: Acd 1096/073/074).

\section{Study procedure}

We retrieved the individual patients' handwritten clinical record files from the record section of the BPKIHS. The data regarding the number of patients, identification no. of patients were derived from the electronic record-keeping system of the Institute which was kept as per international classification of disease coding. The permission to retrieve the files was taken from the Head of the Department of Internal Medicine, Head of the department of anesthesiology and critical care, and Hospital director of the B.P. Koirala Institute of Health Sciences. Data were collected as per the details required by case record form that included the age, sex, address, history, clinical characteristics, and outcome of the patients. Clinical characteristics included symptoms profile, grading of symptoms, the onset of symptoms, mode of injuries, the material of injuries, and immunization status. Blood pressure, respiratory rate, pulse rate, temperature, and saturation of oxygen during triaging at the emergency room, and initial laboratory test reports were recorded once only at baseline. The outcome of patients was recorded as survival, mortality, leave against medical advice (LAMA), and referral to another center.

\section{Data analysis}

Data from the case records file were first recorded in Proforma and then filled into Microsoft Excel 2010. It was analyzed by using IBM SPSS version 20. For descriptive analysis, frequency, percentage, mean, median, standard deviation, and the interquartile range were calculated. For inferential statistics outcomes were divided into favorable outcomes (i.e. patient survival) and unfavorable outcomes (i.e. patient death, who went on LAMA, referred patient)

\section{Results}

Forty patients were included in the study. The study population's mean age was 47 years ( SD-16) and mostly consisted of males $(95.0 \%)$ who had not been immunized with tetanus toxoid in the past (67.5\%) (Table 1).

Punctured wound (45.0\%) followed by an incisional wound (20.0\%) was the common type of skin breach. The most common type of material causing a wound was a wood stick (35\%). Upper or lower limb injuries were most frequent. Seventeen patients (42.5\%) had Ablett grade 4 generalized tetanus. Tracheostomy was performed in 30 patients $(75.0 \%)$ and 24 patients $(60.0 \%)$ had to be mechanically ventilated during the hospital stay. Twentynine out of the 40 patients survived (72.5\%, Fig.1). The in-hospital mortality rate was $7.5 \%$, seven patients $(17.5 \%)$ were discharged against medical advice and one patient $(2.5 \%)$ was referred to another center.

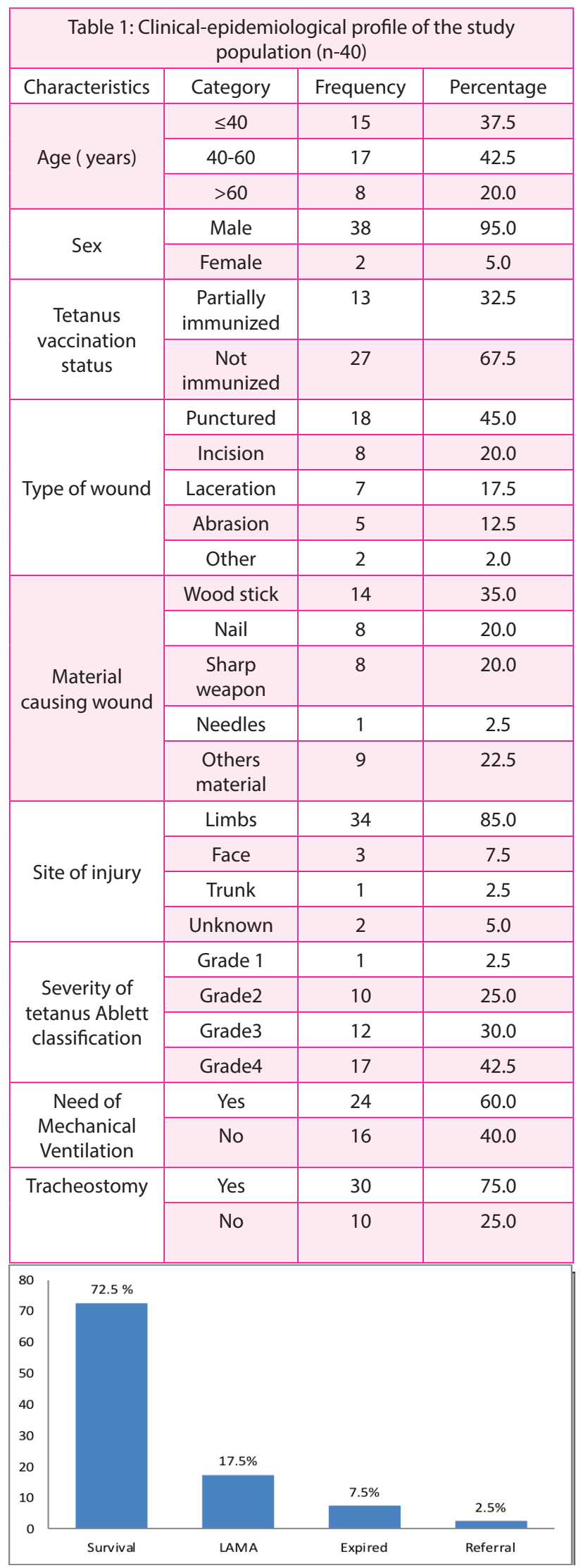

Figure 1: Percentage of the outcome of patients (N-40) with Generalized tetanus after hospital admission; LAMA: Leave against medical advice 


\section{Discussion}

In this retrospective study of 40 adult patients with generalized tetanus, we found that majority of generalized tetanus were above 40 years of age $(62.5 \%)$, male gender $(95 \%)$, and not immunized $(67.5 \%)$. The commonest type of wound and site of the wound were punctured wounds and limbs respectively. Most of the patients presented with Ablett grade 3 or 4 diseases and needed a tracheostomy (75\%) for ventilation. Twenty-nine patients (72.5\%) were discharged from the hospital after completion of treatment.

In this study, we reported that generalized tetanus predominantly affects the male gender. Similarly, the finding of male dominance was reported in Nigeria and Bangladesh. ${ }^{11-12}$ This finding of male predominance might be due to the successful launch of maternal neonatal tetanus elimination programs in these countries and the greater exposure of males to outdoor activities.

Of 40 patients with generalized tetanus, most of the patients $(62.5 \%)$ were aged 40 years and over. The mean age of generalized tetanus patients reported by Obiabo $\mathrm{Y}$ from Nigeria was $34.07 \pm 1.50$ years 11 and Chalya PL et al was $36.21+14.64$ years. ${ }^{13}$ This increase in the prevalence of non-neonatal tetanus with an increase in age may reflect a decrease in the protective level of antibodies with age..$^{14}$ In our study, minor wounds like a punctured wound, incisional wound, and abrasion occurred in $77.5 \%$ of patients. Our finding corroborates with the report of Marulappa VG et al where acute injuries like prick, abrasion, and laceration occurred in $47.9 \%$ of generalized tetanus patients.4 The most common materials inflicting the wound in our study were wood sticks (35\%), nails (20\%), and sharp weapons (20\%). Farming is the main occupation in Nepal, and injuries form materials like wood sticks or nails during the outdoor activities were common and often neglected. Although puncture wounds, incision wounds or abrasions may look benign, such injuries should thus be managed with utmost care to reduce the risk of tetanus.

In our study, 30 patients (75\%) underwent tracheostomy and 24 patients $(60 \%)$ underwent mechanical ventilation at some point during their stay. Similarly, a study in India reported that $74 \%$ of patients needed a tracheostomy, and $45 \%$ needed mechanical ventilation. ${ }^{15}$ However, in a retrospective study conducted among patients with generalized tetanus at Jimma university teaching hospital Southwest Ethiopia, tracheostomy and mechanical ventilation were performed only in $10.5 \%$ and $11 \%$ of patients, respectively. ${ }^{16}$ This lower rate of tracheostomy and mechanical ventilation may reflect differences in the availability of facilities and trained personnel between these countries. Our findings suggest that many patients with generalized tetanus will at some point need mechanical ventilation.

In terms of outcomes, $72.5 \%$ of the patients (29/40) were discharged after treatment, $17.5 \%$ (7/40 patients) left the hospital against the medical advice, $7.5 \%$ (3/40 patients) died during the hospital stay and $2.5 \%$ (1/40 patient) were referred to another center, the reason for which was unknown. The survival rate of generalized tetanus is this study is greater than the survival rate of generalized tetanus reported by Edmondson RS et al. (57.8\%) ${ }^{3}$ and Trujillo $\mathrm{MH}$ et al. (33.6\%). ${ }^{17}$ This may reflect the availability of intensive care facilities and personnel trained to a standardized treatment protocol. The proportion of patients who had left against medical advice in our study (17.5\%) was higher than reported by Edmonston RS et al (4.1\%). ${ }^{3}$ This finding may be due to differences in financial status, and patients' next of kin perception of the disease. The hospital-based case fatality rate in this study (7.5\%) was similar to findings reported by Edmonston RS et al $10 \%{ }^{3}$ and lower than that reported by Amare A et al $(38.0 \%)^{16}$, Chukwubike OA et al $(42.9 \%)^{7}$ and Chalya PL et al (43.9\%). ${ }^{18}$ The lower hospital case fatality in our study may reflect the availability of an intensive care facility in the hospital. This study is the first one to provide data on the epidemiology, mode of injuries, and outcome of generalized tetanus in adult population of eastern region of Nepal. This was a monocentric retrospective study and may not represent the data of generalized tetanus of the country. The data regarding the details of the treatment, the motivation for LAMA, and the outcome of patients who had undergone LAMA and referral was not available. We recommend a multicentric prospective study to determine the current status of tetanus in Nepal and assess the effectiveness of various therapeutics in generalized tetanus.

\section{Conclusion}

Generalized tetanus is still a public health concern in Nepal. It overwhelmingly affects males over 40 years, who present commonly with acute injuries such as punctured, abrasion from wood stick or needle. Most of the patients need a tracheostomy and mechanical ventilation. The in-hospital case-fatality rate was $7.5 \%$.

\section{Acknowledgments}

We Acknowledge Prof. Denis Jaboudon from the University of Geneva for his contribution to manuscript editing.

1. Kyu HH, Mumford JE, Stanaway JD, et al. Mortality from tetanus between 1990 and 2015: findings from the global burden of disease study 2015. BMC Public Health. 2017;17(1):179. doi:10.1186/s12889-017-4111-4

2. Tetanus | Causes and Transmission | Lockjaw | CDC. https:// www.cdc.gov/tetanus/about/causes-transmission.html. Accessed January 13, 2018.

3. Edmondson RS, Flowers MW. Intensive care in tetanus: management, complications, and mortality in 100 cases. $\mathrm{Br}$ Med J. 1979;1(6175):1401-1404. doi:10.1136/BMJ.1.6175.1401

4. Marulappa VG, Manjunath R, Mahesh Babu N, et.al. A Ten Year Retrospective Study on Adult Tetanus at the Epidemic Disease (ED) Hospital, Mysore in Southern India: A Review of 512 Cases. J Clin Diagn Res. 2012;6(8):1377-1380. doi:10.7860/ JCDR/2012/4137.2363

5. Sathirapanya P, Sathirapanya C, Limapichat K, et.al. Tetanus: a retrospective study of clinical presentations and outcomes in a medical teaching hospital. J Med Assoc Thail. 2009;92(3):315319. http://www.ncbi.nlm.nih.gov/pubmed/19301722. Accessed October 6, 2017.

6. Hesse IFA, Asante DK, Lartey M, et.al. Characteristics of adult tetanus in Accra. WAJM. 2003;22(4):291-294.

7. Chukwubike OA, God'spower AE. A 10-year review of outcome of management of tetanus in adults at a Nigerian tertiary hospital. Ann Afr Med. 8(3):168-172. doi:10.4103/15963519.57239

8. Anuradha S. Tetanus in Adults - A Continuing Problem : An Analysis of 217 Patients Over 3 Years from Delhi, India, with Special Emphasis on Predictors of Mortality. Med J Malaysia. 2006;61(1):7-14.

9. Vandelaer J, Partridge J, Suvedi BK,et al. Process of neonatal tetanus elimination in Nepal. J Public Health (Bangkok). 2009;31(4):561-565. doi:10.1093/pubmed/fdp039

10. Bhattarai A. In patient services. bpkihs.edu. http://bpkihs.edu/ hospital-services/in-patient-services.html. Published 2017. Accessed October 12, 2017. 
11. Obiabo Y. Clinical profile and outcome of adult tetanus in Oghara, Delta State, Nigeria. Neurology. 2016;86(16 Supplement).http://n.neurology.org/content/86/16 Supplement/P1.309.

12. Hossain I. et al. Outcome of tetanus Patients admitted in Rangpur Medical College Hospital. BSMMU J. 2014;7(2):77-83.

13. Lisboa T. et al. Guidelines for the management of accidental tetanus in adult patients. Rev Bras Ter Intensiva. 2011;23(4):394409.

14. Sanford JP. Tetanus — Forgotten but Not Gone. N Engl J Med. 1995;332(12):812-813. doi:10.1056/NEJM199503233321209

15. Sidhartha SS, Peter JV, Subhash HS, et al. A proposed new scoring system for tetanus. Indian J Crit Care Med. 2004;8:168172.

16. Amare A, Yami A. Case-fatality of adult tetanus at Jimma University Teaching Hospital, Southwest Ethiopia. Afr Health Sci. 2011;11(1):36-40. http://www.ncbi.nlm.nih.gov/ pubmed/21572855. Accessed January 15, 2018.

17. Trujillo MH, Castillo A, España J, et.al. Impact of intensive care management on the prognosis of tetanus. Analysis of 641 cases. Chest. 1987;92(1):63-65. http://www.ncbi.nlm.nih.gov/ pubmed/3595250. Accessed January 18, 2018.

18. Chalya PL, Mabula JB, Dass RM,et.al. Ten-year experiences with Tetanus at a Tertiary hospital in Northwestern Tanzania: A retrospective review of 102 cases. World J Emerg Surg. 2011;6:20. doi:10.1186/1749-7922-6-20 